\title{
Identification of the flow pattern of liquid streams in the shell-side of a segmental-baffled shell-and-tube heat exchanger
}

Grzegorz LIGUS, Daniel ZAJĄC, Szymon KOŁODZIEJ

DOI: $10.30464 /$ jmee.2018.2.3.245

Cite this article as:

Ligus G., Zając D., Kołodziej S. Identification of the flow pattern of liquid streams in the shell-side of a segmentalbaffled shell-and-tube heat exchanger. Journal of Mechanical and Energy Engineering, Vol. 2(42), No. 3, 2018, pp. 245-256.

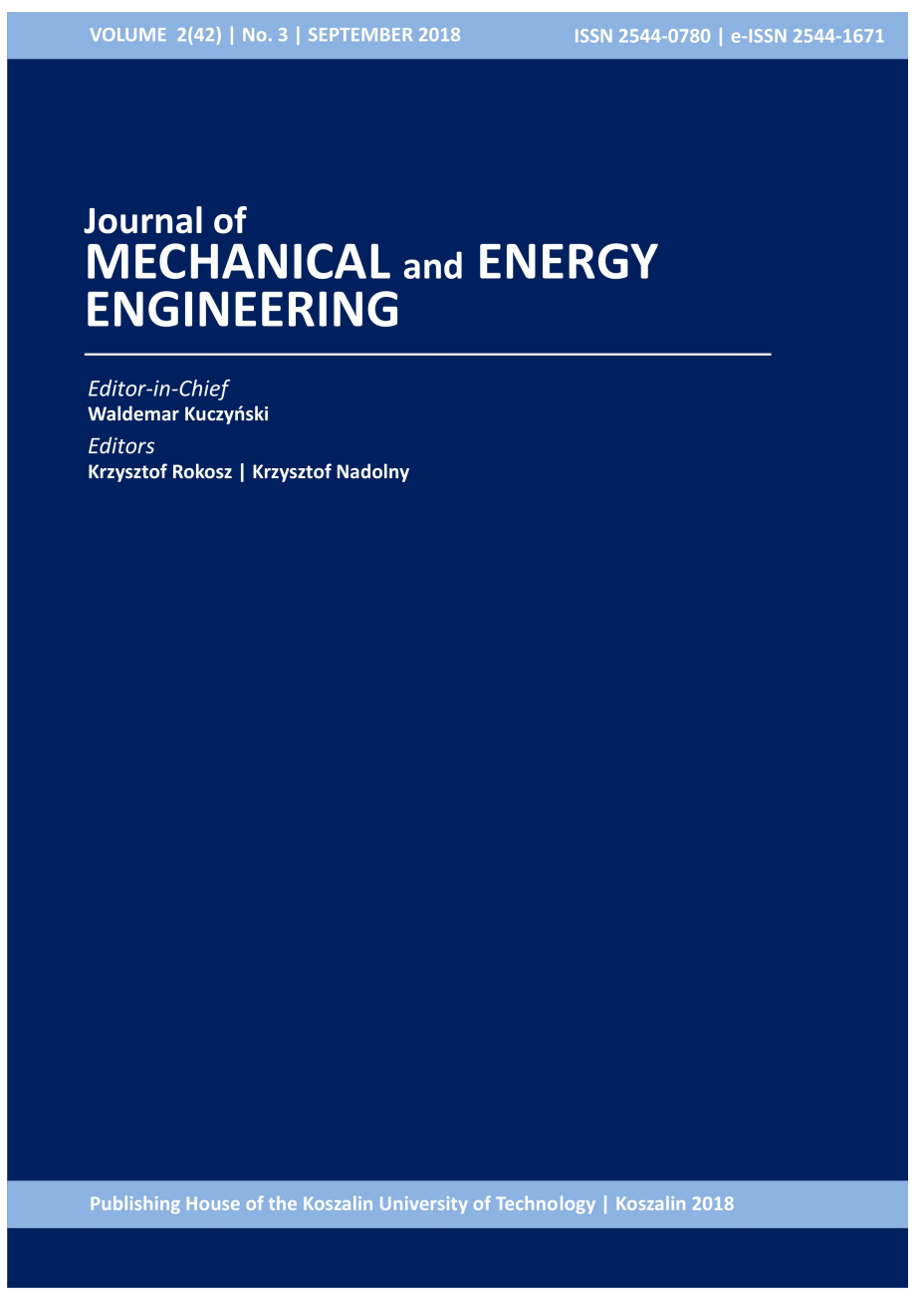

Journal of Mechanical and Energy Engineering

Website: jmee.tu.koszalin.pl

ISSN (Print): 2544-0780

ISSN (Online): 2544-1671

Volume: 2(42)

Number: 3

Year: 2018

Pages: 245-256

Article Info:

Received 13 August 2018

Accepted 17 September 2018

\section{Open Access}

This article is distributed under the terms of the Creative Commons Attribution 4.0 (CC BY 4.0) International License (http://creativecommons.org/licenses/by/4.0/), which permits unrestricted use, distribution, and reproduction in any medium, provided you give appropriate credit to the original author(s) and the source, provide a link to the Creative Commons license, and indicate if changes were made. 


\title{
IDENTIFICATION OF THE FLOW PATTERN OF LIQUID STREAMS IN THE SHELL-SIDE OF A SEGMENTAL-BAFFLED SHELL-AND-TUBE HEAT EXCHANGER
}

\author{
Grzegorz LIGUS $^{1 *}$, Daniel ZAJĄC ${ }^{2}$, Szymon KOŁODZIEJ ${ }^{3}$ \\ ${ }^{1 *}$ Faculty of Mechanical Engineering, Department of Environmental Engineering, Opole University of \\ Technology, Opole, Poland, e-mail: g.ligus@po.opole.pl \\ ${ }^{2}$ Kelvion Sp. z o. o. Opole, Poland, e-mail: d.zajac@po.opole.pl \\ ${ }^{3}$ Faculty of Mechanical Engineering, Department of Vehicles, Opole University of Technology, Opole, \\ Poland, e-mail: s.kolodziej@po.opole.pl
}

(Received 13 August 2018, Accepted 17 September 2018)

\begin{abstract}
Shell-and-tube heat exchangers are widely used in chemical and process engineering. Assessing hydrodynamics of fluid flow in their shell-side is a highly complex task. This results from the complex geometry of the shell-side itself, defined by such parameters as the tubesheet layout, tube diameter, baffle spacing or baffle cut. Shell-and-tube heat exchangers are the subject of many studies in which design and flow parameters are analysed. However, only a few studies concentrate on issues strictly related to the identification of streams of the liquid flowing in the shell-side of apparatus on an industrial scale. In this article, the authors present the results of an experimental visualization study, utilizing Particle Image Velocimetry (PIV). The experiment used a laser sheet technique along and across a tube bundle. The main results of the measurements and analyses concentrate on identifying the flow pattern of streams in the shell-side and assessing stagnation vortices and their consequences. Finally, detecting bypass streams and leakage streams flowing through design gaps between the shell and the tube bundle as well as between the baffles and the tubes in the bundle are presented.
\end{abstract}

Keywords: PIV, shell-and-tube, tube bundle, leakage stream, bypass stream

\section{INTRODUCTION}

Shell-and-tube heat exchangers are one of the main types of equipment used in industry for heat transfer. In order to increase their efficiency, design modifications in the form of introducing baffles to the shell-side are frequently employed [21, 24, 26]. Baffles are meant to increase the flow turbulence of the liquid swilling the tube bundle and thereby increase the heat transfer coefficient. There are several types of baffles (segmental [5, 25], rod [1], disc and doughnut [16], helical [15-17, 22, 23] and other [14]) differing in hydrodynamic characteristics, practical considerations or manufacturing costs. Among the mentioned baffle types, segmental baffles provide high application flexibility combined with a large increase in the heat transfer coefficient $[1,13,24]$. However, large pressure drops generated by disrupted flow in the shell-side constitute a downside to their use. This directly results in increased operating costs of the heat exchanger. Furthermore, choosing incorrect baffle spacing or baffle cut may lead to high variations in local liquid flow velocities. The vortex area which then forms, generates additional local flow resistance [2]. Unfavourable design and flow conditions may cause a vortex area to develop into a permanent stagnation zone characterized by an average flow velocity much below the assumed design value. Operating the shall-and-tube heat exchanger under such conditions considerably lowers its efficiency [3]. Complete knowledge concerning the flow of the agent in the shell-side, on which optimal baffle placement may be based (a compromise between increased pressure drops and expected high heat transfer 
coefficient), is therefore of utmost importance. Particle Image Velocimetry (PIV) is one of the methods which allow to make such an assessment [3-6, 12, 18-20]. It is a non-invasive optical measurement method allowing for visualization of the actual movement of the liquid in the shell-side.

\section{EXPERIMENTAL INVESTIGATIONS}

Using the PIV method, tests were carried out whose aim was to determine liquid pattern in the shellside of the shell-and-tube heat exchanger. The spatial imaging of the phenomena observed required research for the same flow parameters in two imaging planes: along a tube bundle and across a tube bundle. A model of the heat exchanger was created with the maximum transparency of the shell-side and the tube bundle. These properties were achieved through the creation a heat exchanger out of Poly(methyl 2methylpropenoate) (PMMA). Imaging along the tube bundle required an optical system for minimizing aberrations in the form of barrel distortions (curvilinear distortions of straight segments of the imaged measurement area) These distortions were caused by the cylindrical surface of the shell of the heat exchanger. Minimization of the aberrations was ensured by a flat-surface optical attachment mounted outside of the shell of the heat exchanger and filled with the same fluid which flows in the shell side. A similar solution was used in the study [6]. The experimental heat exchanger consisted of a shell with an internal diameter of $240 \mathrm{~mm}$ and the length of 2000 $\mathrm{mm}$ as well as a bundle of 37 tubes with a diameter of $20 \mathrm{~mm}$. A square tube layout with a pitch of $30 \mathrm{~mm}$ was chosen for the study. Such a geometric layout allowed an introduction of a light sheet between the tubes in the case of long tube imaging. Ten segmental baffles with a cut of $25 \%$ were installed on the tube bundle. In order to eliminate the gas phase from the system, a ventilation valve was installed in front of the base of the first baffle. The described model of the heat exchanger was the main component of the experimental stand. The configurations of the experimental stand was dependent on the imaging plane. Both sets are shown in Figure 1.

Water was the liquid circulating in the shell-side; it was stored in a tank with a capacity of $200 \mathrm{dm}^{3}$. A multistage pump controlled via signals from an electromagnetic flowmeter was responsible for the circulation of the liquid through the exchanger. The utilized experimental method required an addition of a seeding particles to the circulating liquid. Seeding particles have to reflect the light of the light sheet of ten grams of Dialuminium trioxide $\left(\mathrm{Al}_{2} \mathrm{O}_{3}\right)$, which is characterized by high reflectivity and guarantees a sufficient amount of reflected light for flow imaging, was used as seeding particles in the presented study. In order to maintain constant homogenization of water and seeding particles, an agitator was installed in the tank. The type and quantity of seeding particles were decided on the basis of an overview of literature [7] and the authors' tests. Hydrodynamics of the heat exchanger model was assessed for two volume of liquid equal to $5 \mathrm{~m}^{3} / \mathrm{h}$ and $10 \mathrm{~m}^{3} / \mathrm{h}$.

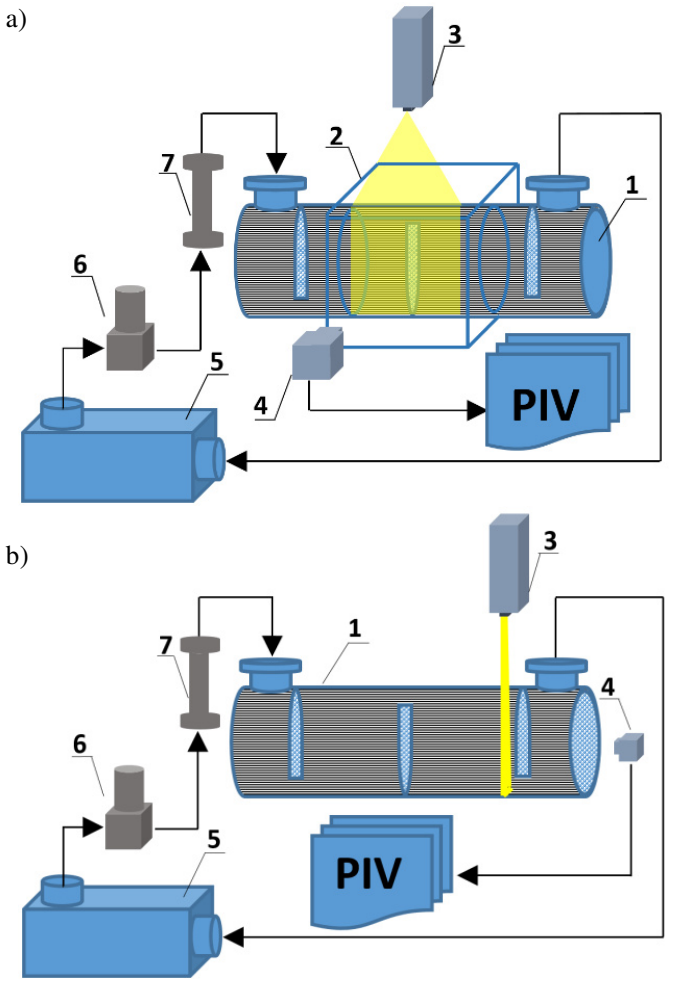

Fig. 1. Experimental stand: imaging along a tube bundle (a), imaging across a tube bundle (b): shell-and-tube heat exchanger model (1), flat-surface optical attachment (2), laser (3), CCD camera (4), tank (5), pump (6), flowmeter (7)

A Dantec Dynamics FlowSense EO-4M CCD camera, enabling orthogonal imaging of the shell-side in both plane. Studies along the tube bundle were performed using single-image imaging. Studies across the tube bundle required multi-position imaging. Positions of the local points during the tests is shown in Figure 2. Such a behaviour was caused by the occurrence of the perspective effect (Fig. 3). Each measurement series in multi-position imaging consisted of nine sections for which PIV analysis was performed. The geometrical combination of the obtained results enabled gaining a full visualization of the flow across the tube bundle. Recording was performed at a resolution of $2048 \times 2048$ pixels in double frame mode at a frequency of $10 \mathrm{~Hz}$. Time between pulses was $1500 \mu$ s for the volume of liquid equal to $5 \mathrm{~m}^{3} / \mathrm{h}$ and $500 \mu \mathrm{s}$ for volume of liquid equal to $10 \mathrm{~m}^{3} / \mathrm{h}$. Each measurement series consisted of 250 double frame images. 


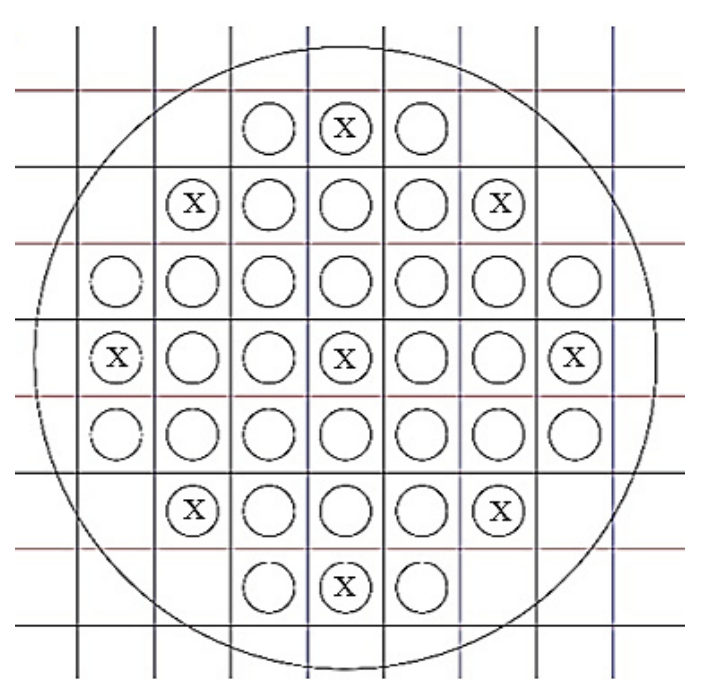

Fig. 2. Positions of the focal point (marked as "x") during across a tube bundle imaging

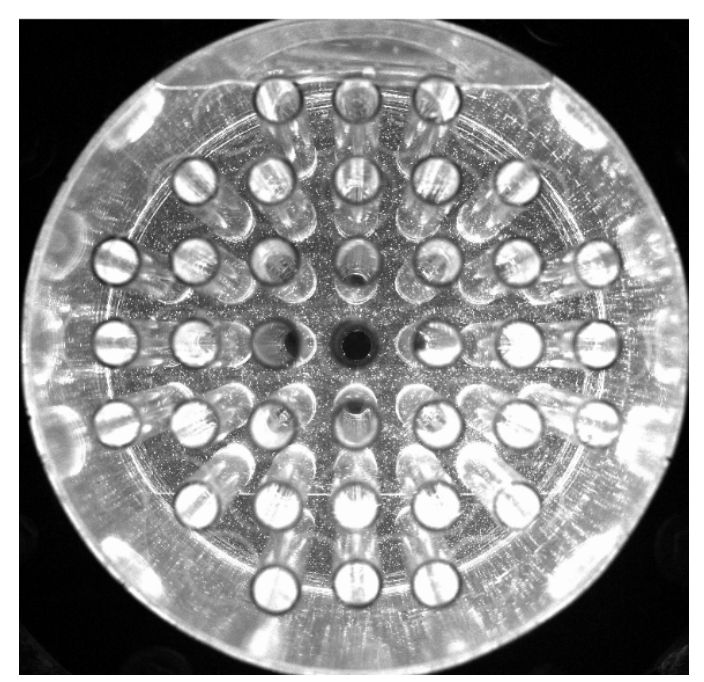

Fig. 3. Effect of the perspective in the shell-and-tube heat exchanger during across tube bundle imaging

The observed area of the shell-side was lit by light from a Dantec Dynamics DualPower TR Nd:YAG laser, utilizing the light sheet technique. The laser generated a coherent light beam with a length of 532 $\mathrm{nm}$ and power of $1200 \mathrm{~mJ}$. During the study, along a tube bundle, the light sheet crossed the space between the second and third tube in a row. For a study across a tube bundle, the laser sheet illuminated the imaging plane in a distance of $1 \mathrm{~cm}$ from the last baffle. Similar optical paths were used in the following studies $[6,8,9,10,11]$. PIV analysis was performed for each of 250 pairs of recorded double frame images. Calculations were performed using Dantec Dynamics Dynamic Studio software, version 2015b. The phases of methodology of calculations and analyses are presented in Figure 4.

\section{RESULTS AND DISCUSSION}

\subsection{Visualization along the tube bundle}

Visualizations of a vector velocity field are presented in Figure 5 and 6 . When the heat exchanger was fed by volume of liquid equal to $5 \mathrm{~m}^{3} / \mathrm{h}$, the area of reduced flow velocity between the baffles was as high as $50 \%$ of its total surface area. Velocity histogram for zone between the baffles (fig. 7) shows that the average velocity in this area is $0.074 \mathrm{~m} / \mathrm{s}$. This should be considered unfavorable. Considerably reduced flow turbulence in this zone decreases heat removal from the tube bundle. In specific heat exchanger applications, e.g. in chemical industry, the liquid may overheat (in the case of temperature sensitive liquids, an uncontrolled change in its properties may occur). A local decrease in flow velocity also generates operating problems in the form of intensified sediment accumulation. The top surfaces of the tubes located directly behind segmental baffles are particularly vulnerable to intensive accumulation of sediment. Accumulation of vortices (fig. 8) may form a barrier to the full circulation of the agent in the shell-side, persisting for a long time (liquid captured in a vortex changes its position only to a limited extent). For the examined geometric layout of the tube bundle, increasing the flow velocity by increasing the volume of liquid to $10 \mathrm{~m}^{3} / \mathrm{h}$ resulted in a visible reduction of the stagnation area (Fig. 6).

Stagnation areas comprised only $20 \%$ of the area between baffles; at the same time, the average velocity in that area increased to $0.29 \mathrm{~m} / \mathrm{s}$. Figure 9 shows that the area behind the baffle whose base is located in the bottom part of the exchanger (left baffle) is less susceptible to the volume of liquid changes than the area behind the baffle installed in the upper part of the exchanger (the right baffle). In the first case (the area behind the left baffle) in both volume of liquid, similar liquid velocity were noticed. Flow disturbances caused by the stagnation pattern should be eliminated by selecting other baffle spacing or flow parameters of liquid.The above analyses indicate that complying with the recommended design flow parameters is vital for an efficient operation of heat exchangers with baffles. Furthermore, a vortex analysis may constitute a component of the hydrodynamic optimization of shell-and-tube heat exchangers. Similar findings were presented in the study $[3,6]$.

The confirmation of the flow inequality behind baffles is also visible in the shape of the velocity profiles calculated in the symmetry axes of the tube for hydrodynamically similar flow areas (fig. 10 and 11). The differences of the velocity value at the area directly behind the baffle installed in the upper part of the exchanger (tube 7) is approximately two times higher compared to the analogical areas (tube 1) behind the baffle installed in its bottom part. This issue requires further study, as this phenomenon may 


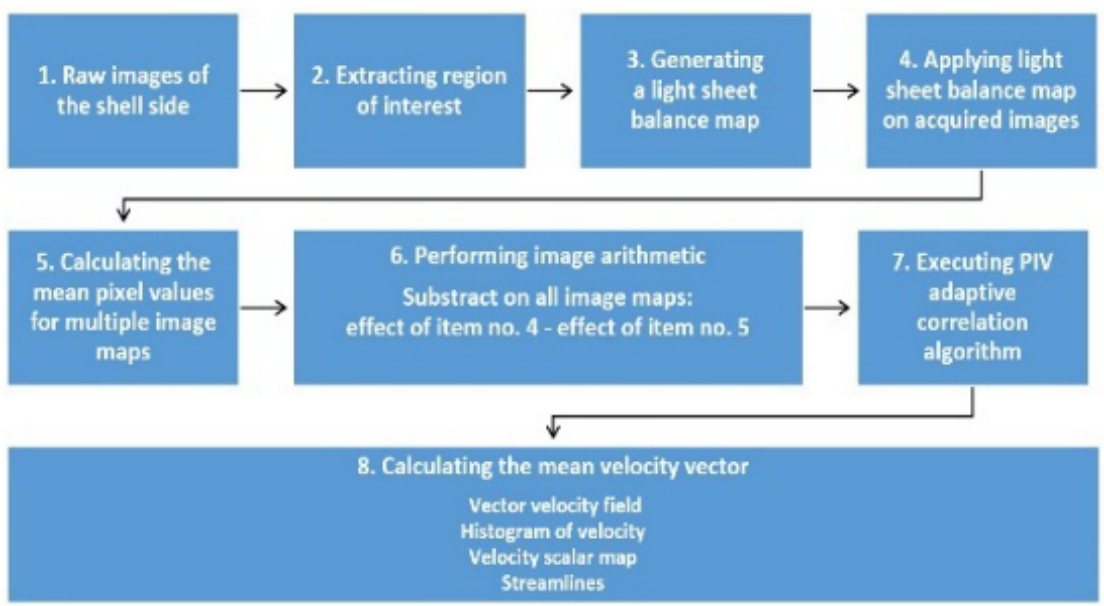

Fig. 4. Applied PIV methodology

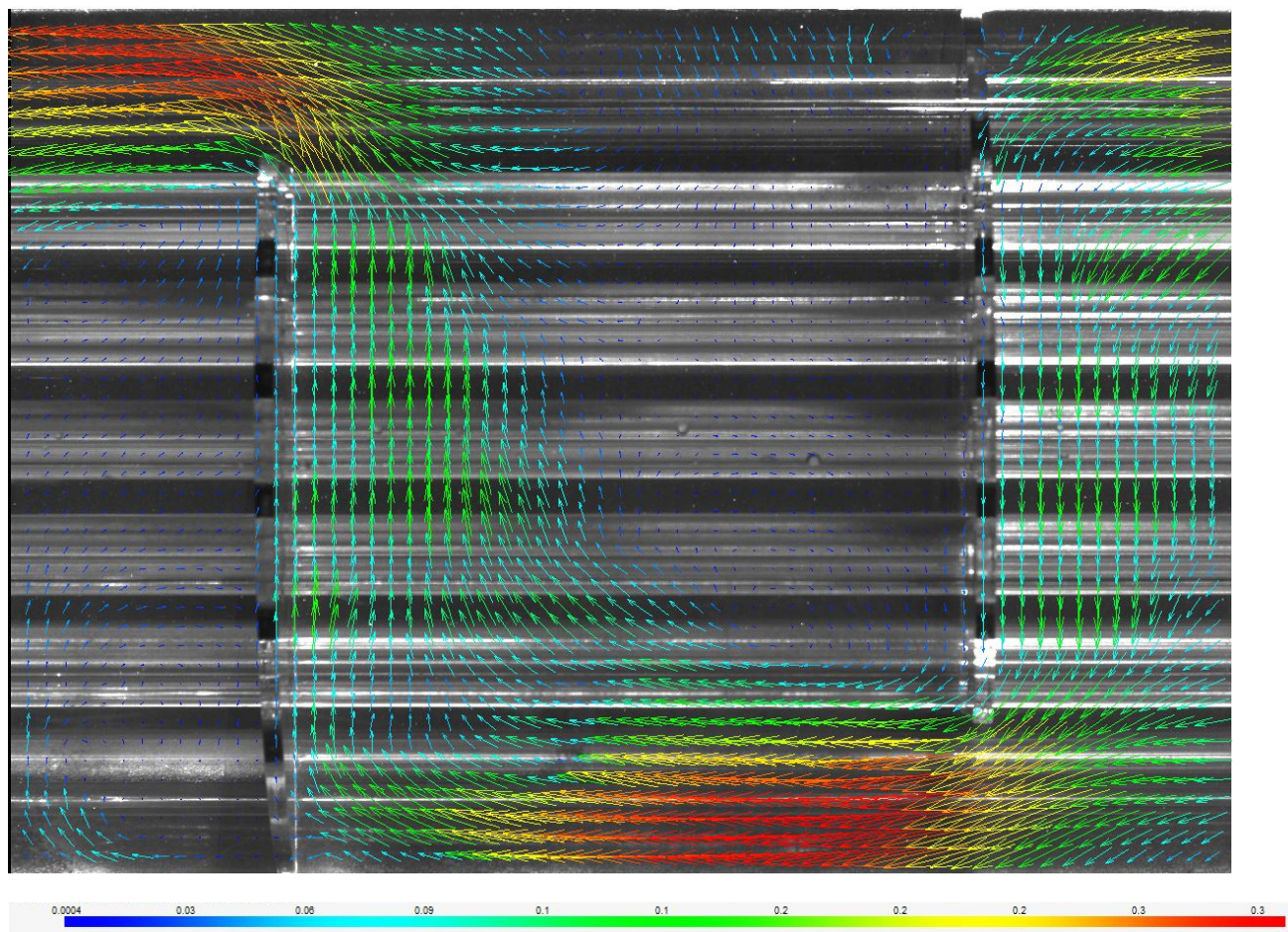

Fig. 5. Vector velocity field in the shell-side of the heat exchanger, volume of liquid equal to $5 \mathrm{~m}^{3} / \mathrm{h}$, velocity in $\mathrm{m} / \mathrm{s}$

determine changes in parameters of heat exchanger operation depending on vertical or horizontal positioning.

Reducing vortex disruptions via increasing liquid flow velocity in the shell-side causes increased the participation of bypass and leakage streams. The visualization of bypass streams are streamlines designated on the basis of the vector velocity field (fig. 10). The intensified presence of bypass streams is clearly confirmed by streamlines that occur in front of the baffles, in the upper and lower part of the shell (area A in Fig. 12). Because in the examined geometric layout, the area between the shell and the external tubes in the tube bundle generates less flow resistance, the areas between each row of tubes, bypass streamlines occur in an increased velocity area. Another characteristic feature of these streamlines is the fact that they are mostly located along $45^{\circ}$ direction lines (the resultant of horizontal and vertical fluid movement in the tube bundle). For the examined geometric layout of the heat exchanger, when the volume of the liquid was equal to $5 \mathrm{~m}^{3} / \mathrm{h}$, bypass streams were incidental. 

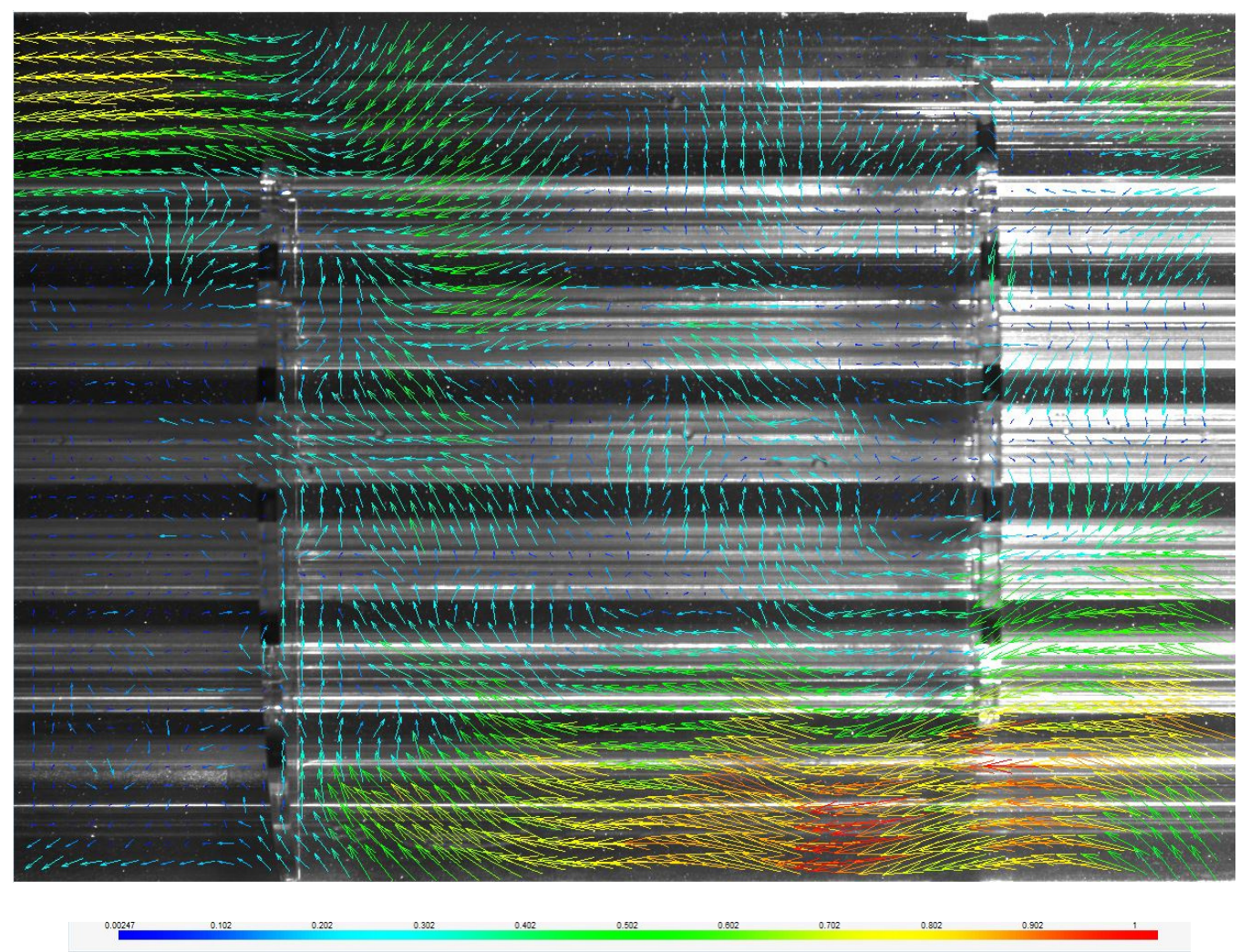

Fig. 6. Vector velocity field in the shell-side of the heat exchanger, volume of liquid equal to $10 \mathrm{~m}^{3} / \mathrm{h}$, velocity in $\mathrm{m} / \mathrm{s}$
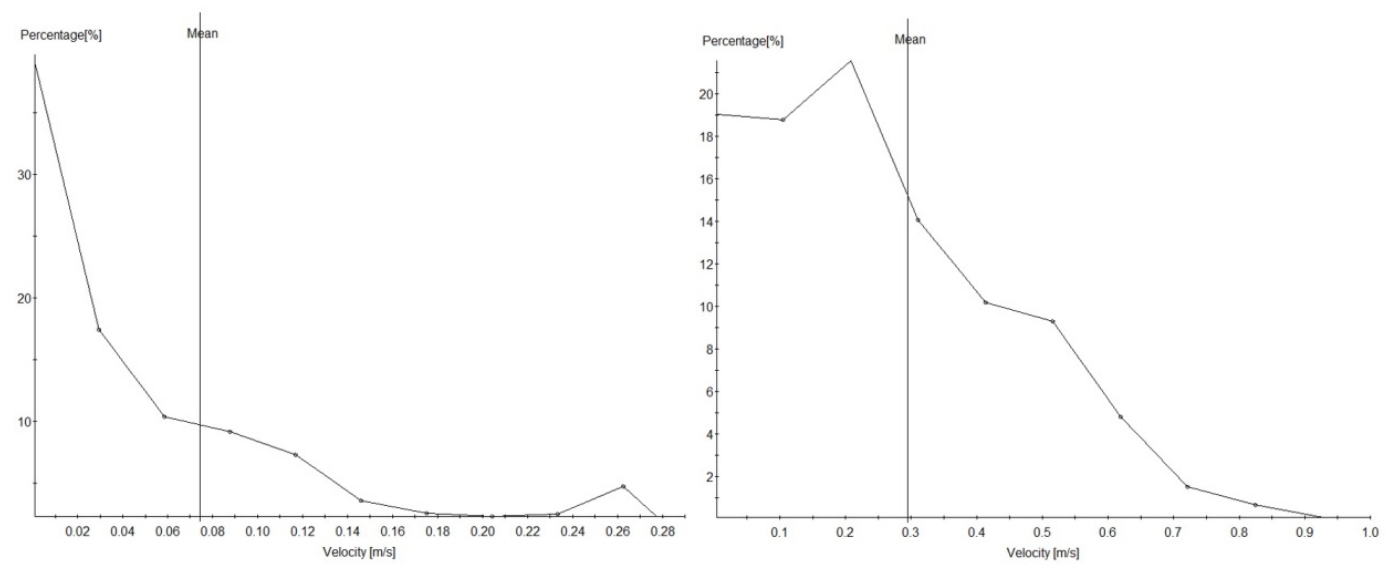

Fig. 7. Histogram of velocity between the baffles: volume of liquid equal to5 $\mathrm{m}^{3} / \mathrm{h}$ (on the left), volume of liquid equal to 10 $\mathrm{m}^{3} / \mathrm{h}$ (on the right)

A visualization study along the tube bundle also enabled an identification of leakage streams flowing between the tubes and the baffles as well as between the edges of the baffles and the shell. Leakage streams may be identified by streamlines crossing the plane of the baffles (area B in Fig. 10). In this area, there is no sudden decrease in velocity to zero at the border marked by the baffle. High local velocity enables easier overcoming of local flow resistances in the gaps, which also manifests on the scalar map by contour lines crossing beyond the plane of the baffle. A similar situation occurs in case of streams flowing through gaps between the edges of the baffles and the shell. Identifying leakage streams is particularly important in the case of installations where high-purity liquids are used (no sediment which would reduce design gaps in time and therefore minimize the participation of leakage streams). 


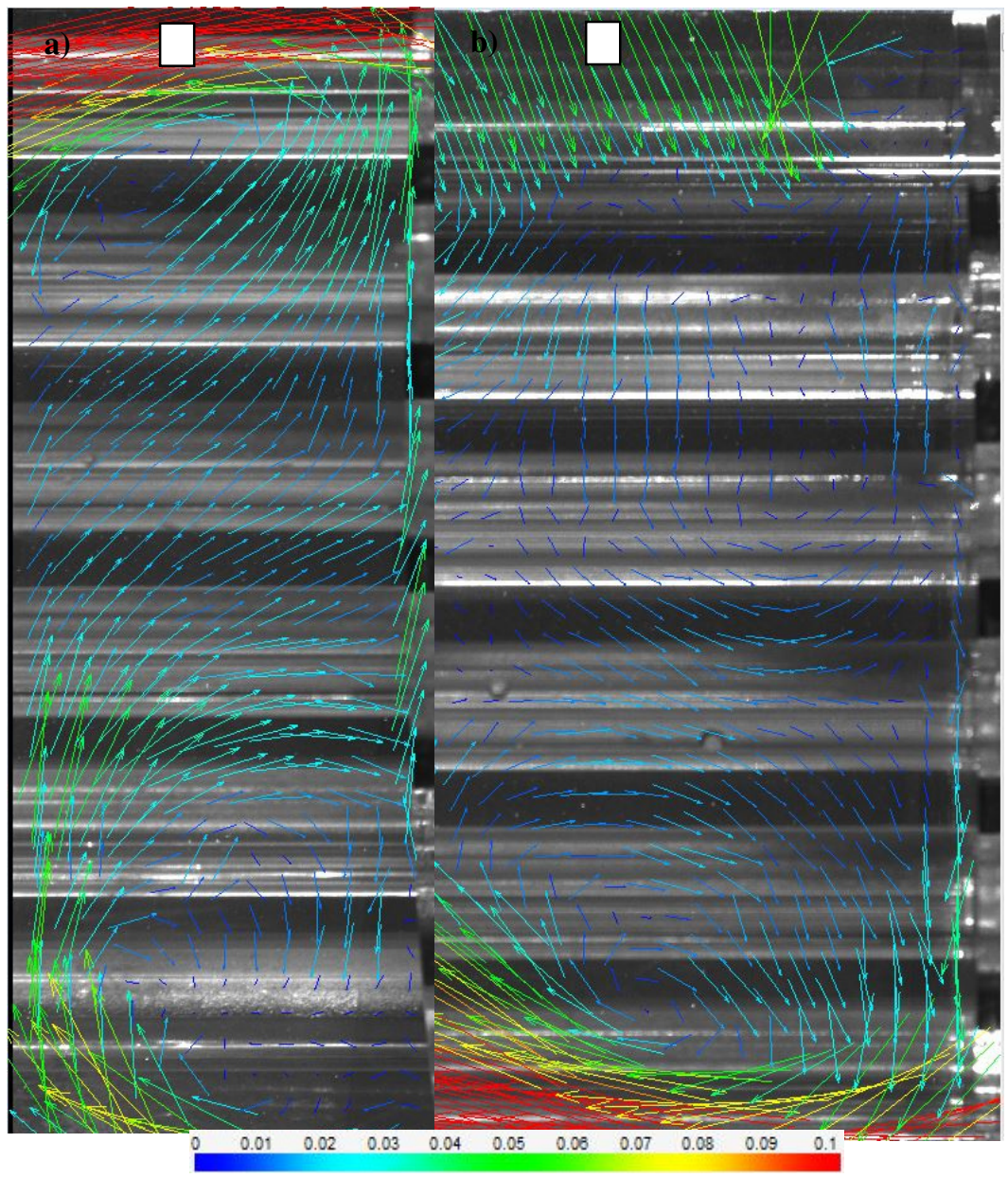

Fig. 8. Stagnation vortices for volume of liquid equal to $5 \mathrm{~m}^{3} / \mathrm{h}$ : left baffle (a), right baffle (b). Velocity in $\mathrm{m} / \mathrm{s}$

\subsection{Visualization across the tube bundle}

In the geometrical assemblies of the obtained liquid, flow visualizations in the shell-and-tube heat exchanger directly ahead the segmental baffle shown in Figures 13 and 14, the stagnation zones can be clearly determined. They occur in the area where the liquid flows around the pipes, below all pipes in the row (hydrodynamic shade of the pipes). In their areas, the velocity decreases significantly to the minimum values. The experiment confirms that as a result of an increase of the flow velocity, the size of the stagnation zones occurring in the hydrodynamic shade of the pipes decreases. In the central part of the shell-side, the stagnation zones have a distribution parallel to the direction of the liquid flow. Moving in the direction of the heat exchanger's shell, one can observe clear oblique deviations, supplying bypass streams on both sides of the heat exchanger. This phenomenon is visibly symmetrical. Bypass streams are also characterized by velocity clearly above the average for a cross-section of the shell-side. The heat exchanger being tested, due to the relative large distance between a tube bundle and shell, tends to generate strong bypass streams. These observations are also reflected in the research along the tube bundle. In the baffle cut zone, a typical turbulent area is formed.

A hydrodynamic nature of baffle cut zone, except for geometrical parameters, is shaped by the flow parameters of the main stream of the liquid and the flow parameters of two bypass streams stirred in the area of the baffle cut zone. As a result of the interaction between the stirring bypass streams, extensive vortex patterns appear in the baffle cut zone. Their influence on the liquid distribution depends on the velocity of the bypass streams. For the volume of the liquid values presented in the study, the area of vortex patterns in the baffle cut zone grew with an increase os the velocity of bypass streams. In addition, in the case of unsteady flow parameters, there may be significant displacements of the cores of individual vortices or entire vortex patterns. Consequently, the symmetrical nature of bypass streams can be disturbed. The interaction of numerous vortices also adversely affects the formation of pressure drops in the heat exchanger. 


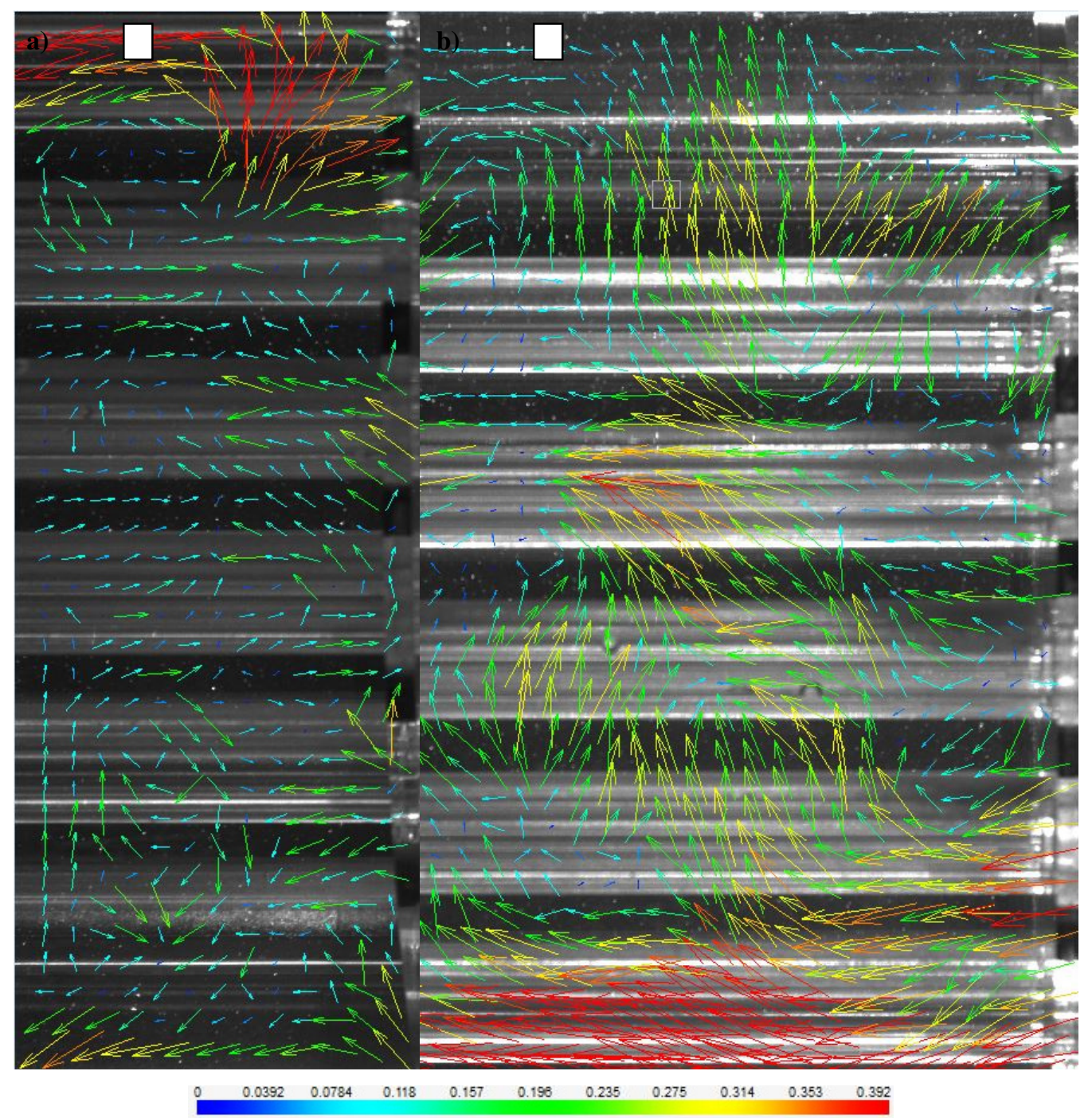

Fig. 9. Stagnation vortices for volume of liquid equal to $10 \mathrm{~m}^{3} / \mathrm{h}$ : left baffle (a), right baffle (b). Velocity in $\mathrm{m} / \mathrm{s}$

Minimalizing of the maldistribution in baffle cut zone can be an important factor in increasing the performance of the shell-and-tube heat exchanger with segmental baffles. A full investigation of the flow pattern of streams across the tube bundle in this type of the heat exchanger requires consideration of the liquid movement towards the imaging plane. For this reason, the StereoPIV technique should be used in the following research.

\section{CONCLUSIONS}

A visualization study was conducted of the hydrodynamics of liquid flow along and across the tube bundle of a heat exchanger with segmental baffles confirmed high effectiveness of PIV technique in the diagnostics of this type of equipment.
The method of identifying stagnation vortices based on a vector analysis may be an effective tool for assessing the operating efficiency of heat exchangers. In the equipment geometry examined, increasing the volume of liquid by $50 \%$ resulted in a decreased participation of stagnation zones by $30 \%$ and tripled liquid average velocity in the shell side between baffles. This change in flow parameters largely overcomes the barrier to the full circulation of the fluid along and across the tube bundle formed by intensive stagnation vortices. The studies confirmed that an increase of the liquid velocity affects a decrease of the area size of stagnation zones. An analysis of velocity profiles revealed an asymmetry of flow conditions for hydrodynamically similar areas behind the baffles installed in the upper and bottom parts of the exchanger. 


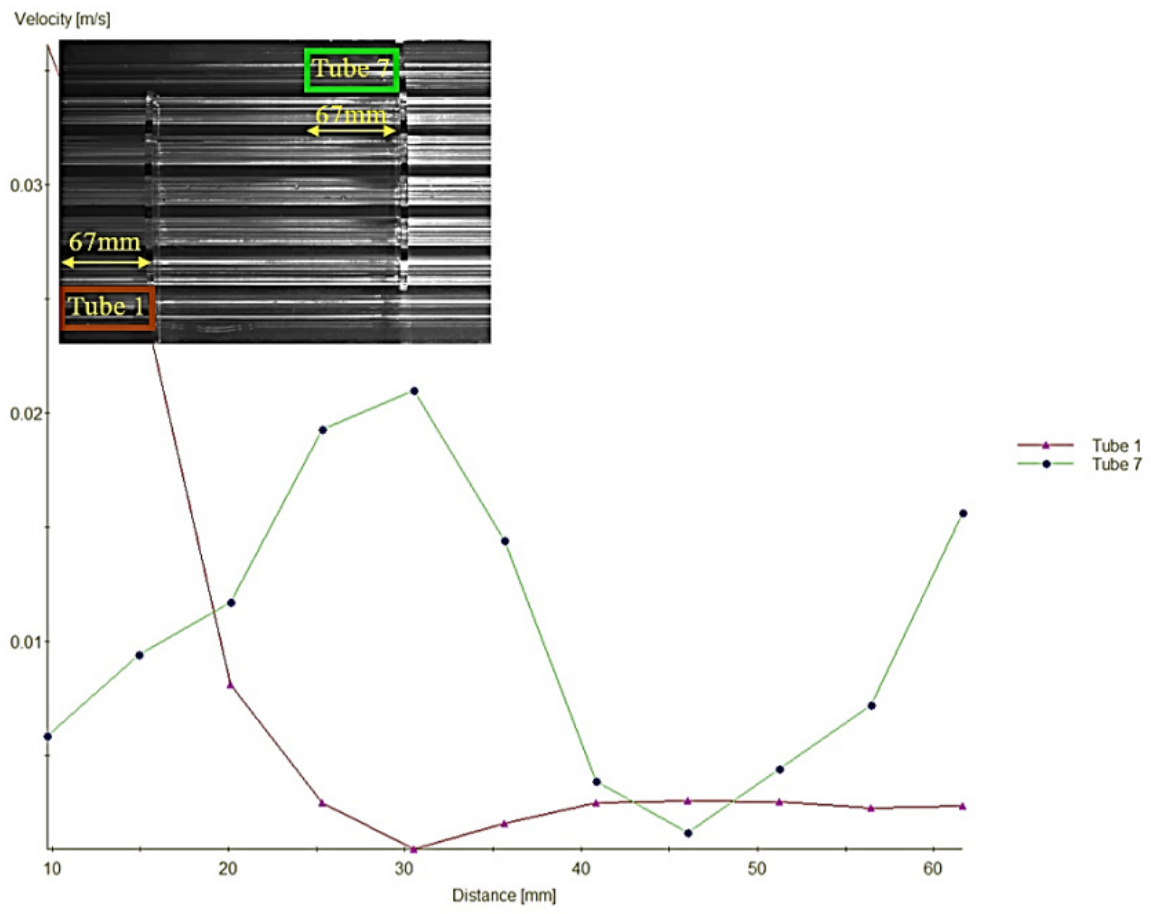

Fig. 10. Velocity profile behind the baffle (volume of liquid equal to $5 \mathrm{~m}^{3} / \mathrm{h}$ )

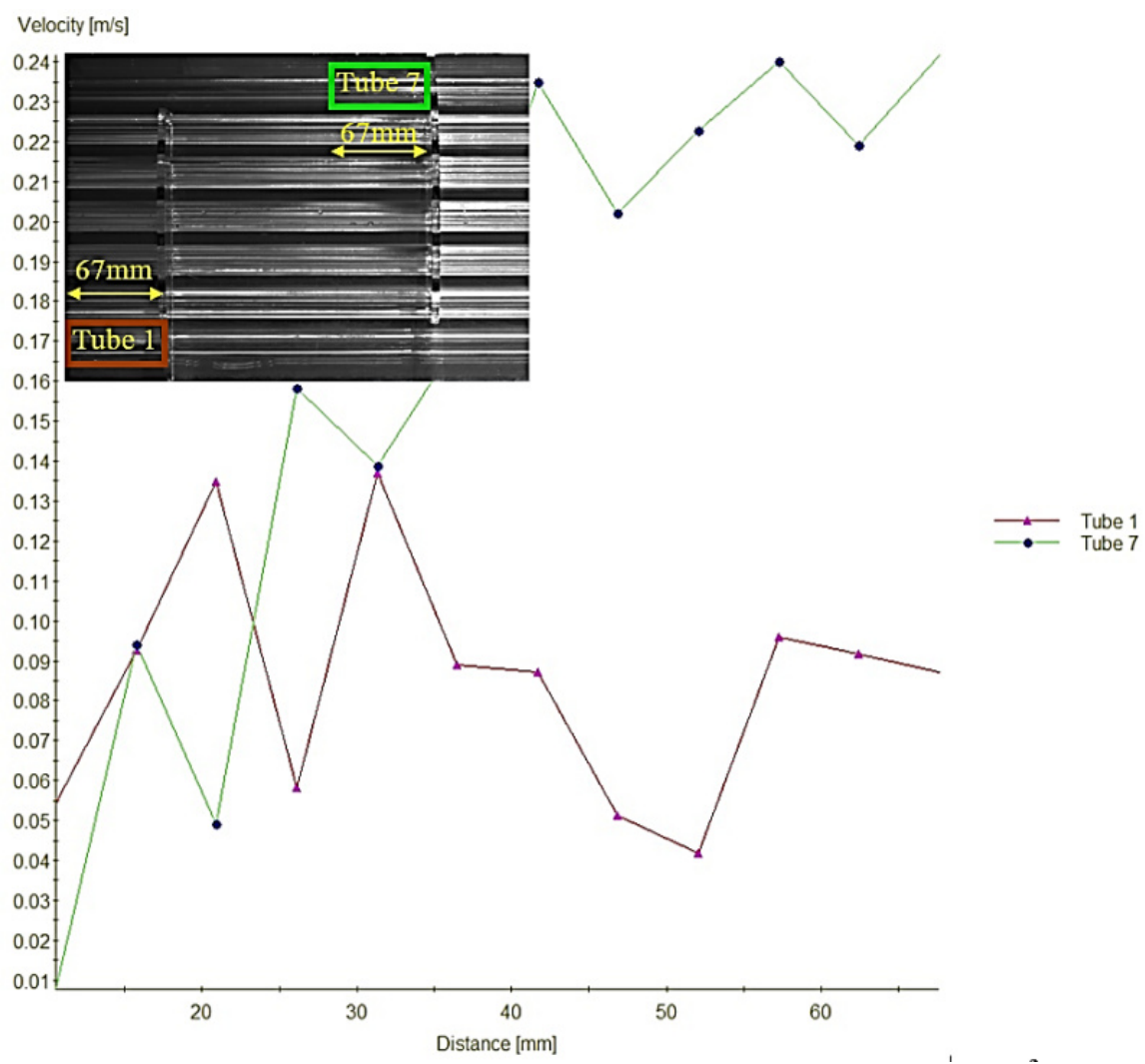

Fig. 11. Velocity profile behind the baffle (volume of liquid equal to $10 \mathrm{~m}^{3} / \mathrm{h}$ ) 


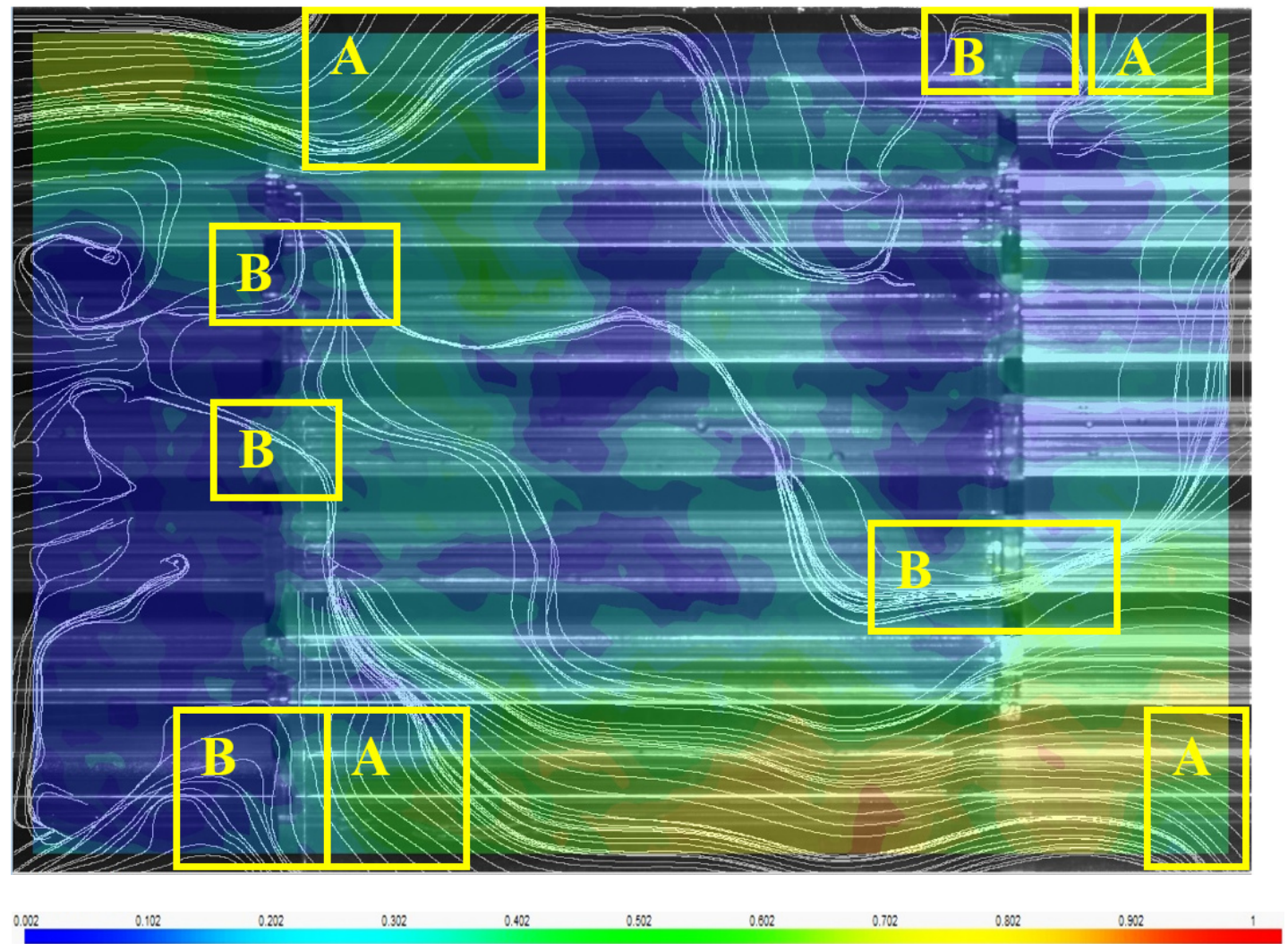

Fig. 12. Identification of the bypass streams (area A) and leakage streams (area B) with a streamlines analysis. Velocity in $\mathrm{m} / \mathrm{s}$

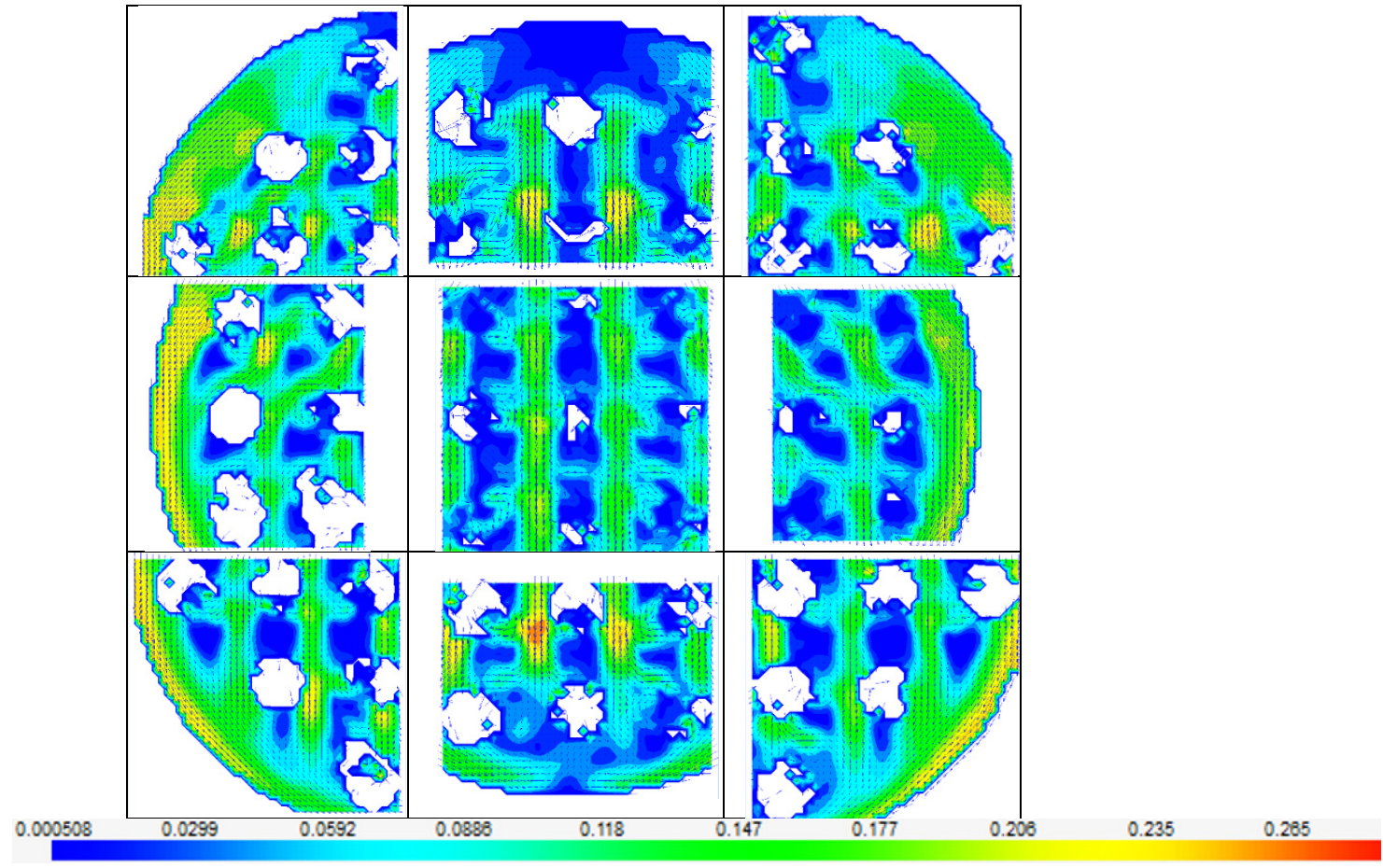

Fig. 13. Visualization of the liquid flow across the tube bundle, volume of liquid equal to $5 \mathrm{~m}^{3} / \mathrm{h}$, velocity in $\mathrm{m} / \mathrm{s}$ 


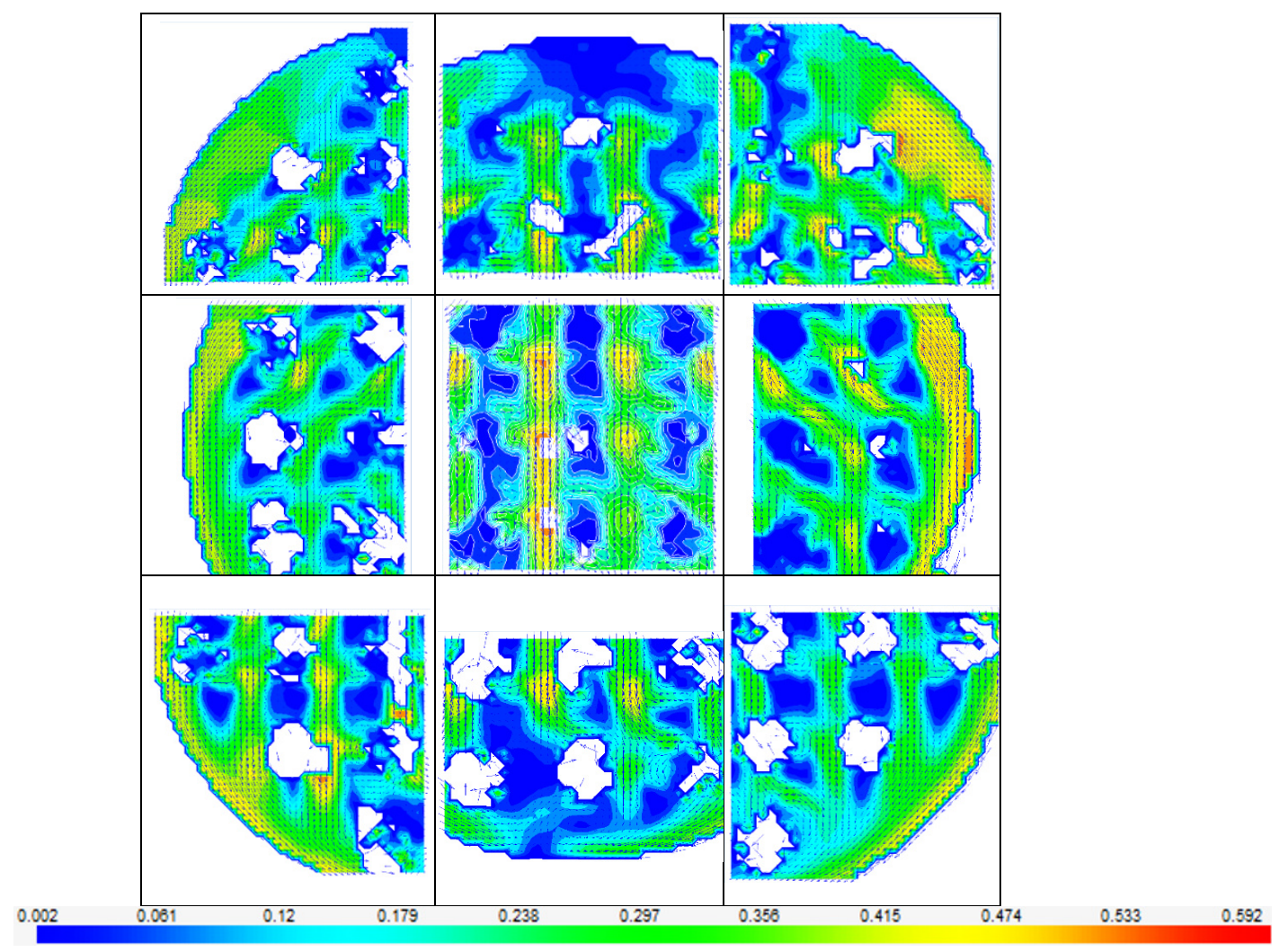

Fig. 14. Visualization of the liquid flow across the tube bundle, volume of liquid equal to $10 \mathrm{~m}^{3} / \mathrm{h}$, velocity in $\mathrm{m} / \mathrm{s}$

Visual research enables an identification of bypass and leakage streams in shell-and-tube heat exchangers. The intensity of bypass streams has a strong impact on the nature of the flow in the baffle cut zone. Issues discussed in the study should be confirmed in a wider research program, especially with the use of the StereoPIV method.

\section{Nomenclature}

\section{Acronyms}

PIV - Particle Image Velocimetry

\section{References}

1. TEMA (2007). Standards of the Tubular Exchanger Manufacturer Association, 9th Edition, New York, USA.

2. Jozaei A.F., Baheri A., Hafshejani M.K., Arad A. (2012). Optimization of baffle spacing on heat transfer, pressure drop and estimated price in a shell-and-tube heat exchanger, World Applied Sciences Journal, Vol. 18 (12),

3. Karaś M., Zając D., Ulbrich R. (2013). Distribution of heat exchange coefficient and vertical two-phase crossflow structures examination over tube bundles, using DPIV and electrochemical methods, Inz. Ap. Chem., 2013, 52, 5, 431-432.

4. Karaś M., Zając D., Ulbrich R. (2014). Experimental investigation of heat transfer performance coe $\square$ cient in tube bundle of shell and tube heat exchanger in twophase flow, Archives of thermodynamics, Vol. 35, No. 1, 87-98, 2014.
5. Guziałowska J., Ligus G., Ulbrich R. (2008). Some problems of flow pattern recognition in complex geometry, Proceedings of 5th International Conference on Transport phenomena in Multiphase Systems, Bialystok, Poland.

6. Chang T.H., Lee Ch., Lee H., Lee K.S. (2015). Velocity profiles between two baffles in a shell and tube heat exchanger, Journal of Thermal Science, Vol. 24, No. 4 (2015) 356-363.

7. Adrian R.J, Westerweel J. (2011). Particle Image Velocimetry, Cambridge University Press, New York, USA.

8. Paul S.S., Tachie M.F., Ormiston S.J. (2007). Experimental study of turbulent cross-flow in a staggered tube bundle using particle image velocimetry, International Journal of Heat and Fluid Flow, Vol. 28, 441-453.

9. Bin Ch., Liejin G. (2000). Particle Image Velocimetry measurement of flow across tube bundle in waste heat boiler, Journal of Thermal Science, Vol.9, No.3, 249256.

10. Velasco F.J.S., Lopez del Pra C, Herranz L.E. (2008). Expansion of a radial jet from a guillotine tube breach in a shell-and-tube heat exchanger, Experimental Thermal and Fluid Science, Vol. 32(4), 947-961.

11. Iwaki C., Cheong K.H., Monji H., Matsui G. (2004). PIV measurement of the vertical cross-flow structure over tube bundles, Experiments in Fluids, Vol. 37, 350-363.

12. Dominguez-Ontiveros E.E., Hassan Y.A. (2009). Nonintrusive experimental investigation of flow behavior inside a $5 \times 5$ rod bundle with spacer grids using PIV and MIR, Nuclear Engineering and Design, Vol. 239, 888898. 
13. Saunders E.A.D. (1983). Features relating to thermal design in Heat exchanger design handbook, Vol. 4, Hemisphere, Section 4.2.5, Washington, USA.

14. Xing L., Zhengpeng M., Sichao T., Ruiqi W., Xiaoyu W. (2018). PIV study of velocity distribution and turbulence statistics in a rod bundle, Annals of Nuclear Energy, Vol. 117 (7), 305-317,

15. Yang S., Chen Y., Wu J., Gu H. (2018). Influence of baffle configurations on flow and heat transfer characteristics of unilateral type helical baffle heat exchangers, Applied Thermal Engineering, Vol. 133, 739-748.

16. Le H., Kottke V. (1999). Analysis of local shellside heat and mass transfer in the shell-and-tube heat exchanger with disc-and-doughnut baffles, International Journal of Heat and Mass Transfer, vol. 42 (13), 3009-3521.

17. Wen J., Yang H., Wang S., Gu X. (2018). PIV experimental investigation on shell-side flow patterns of shell and tube heat exchanger with different helical baffles, International Journal of Heat and Mass Transfer, Vol. 104, 247-259.

18. Lee S., Delgado M., Lee Y., Hassan Y.A. (2018). Experimental investigation of the isothermal flow field across slant 5 -tube bundles in helically coiled steam generator geometry using PIV, Nuclear Engineering and Design, Vol. 338, 261-268.

19. Delgado M., Lee S., Hassan Y.A., Anand N.K. (2018). Flow visualization study at the interface of alternating pitch tube bundles in a model helical coil steam generator using particle image velocimetry, International Journal of Heat and Mass Transfer, Vol. 122, 614-628.

20. Mourad Y., Fayolle F., Legrand J. (2011). Flow patterns analysis using experimental PIV technique inside scraped surface heat exchanger in continuous flow condition, Applied Thermal Engineering, Vol. 31, 28552868.

21. Bell K.J. (2004). Heat exchanger design for the process industries, Journal of Heat Transfer, vol. 126 (2004), pp. 877-885.

22. Saeedan M., Bahiraei M. (2015). Chemical engineering research and design effects of geometrical parameters on hydrothermal characteristics of shell-and-tube heat exchanger with helical baffles: Numerical investigation, modeling and optimization, Chemical Engineering Research and Design, Vol. 96, 43-53.

23. Nemati Taher F., Zeyninejad Movassag S., Razmi K., Tasouji Azar R. (2015). Baffle space impact on the performance of helical baffle shell and tube heat exchangers, Applied Thermal Engineering, vol. 44, 143149.

24. Webb R.L, (1994). Principles of Enhanced Heat Transfer, Wiley, New York.

25. Mellal M., Benzeguir R., Sahel D., Ameur H., (2017). Hydro-thermal shell-side performance evaluation of a shell and tube heat exchanger under different baffle arrangement and orientation, International Journal of Thermal Sciences, Vol. 121, 138-149.

26. Sthlik P. Wadekar V.V. (2002). Different strategies to improve industrial heat exchanger, Heat Transfer Engineering, Vol. 23, 36-48.

\section{Biographical notes}

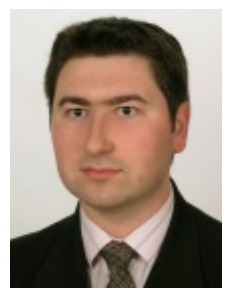

Grzegorz Ligus Ph.D. (Eng.), graduated from the Faculty of Mechanical Engineering of Opole University of Technology (2004) where he had been studying environmental engineering. $\mathrm{He}$ obtained his doctoral degree at Opole University of Technology (2008), his doctoral thesis concerning the field of machine construction and operation. Until 2010, he was working as the Head of the Environmental Engineering Department in the Institute of Ceramics and Building Materials in the Material, Process and Environmental Engineering Division in Opole. Currently, he works as Assistant Professor in the Department of Environmental Engineering of the Faculty of Mechanical Engineering at the Opole University of Technology. Scientific interests: aeroand hydrodynamics in single- and multiphase systems, experimental fluid mechanics.

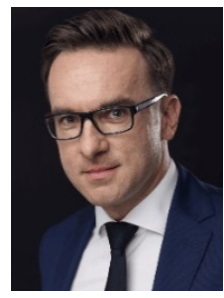

Daniel Zając has received his $\mathrm{PhD}$ in Mechanical Engineering from Faculty of Mechanical engineering in Opole University of Technology in 2004. His specialization is experimental fluid mechanics and thermal processes. For 13 years he was working as an Assistant Professor in Opole University of Technology. Since 2016 he is the Head of Engineering and R\&D Department in Kelvion Sp. z o.o. - the world leader in industrial heat exchangers production.

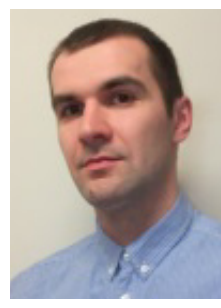

Szymon Kołodziej received a MSc Eng. degree in 2015 after he had completed his Mechnical Engineering studies (in the specialization of Cars and Tractors) at the Faculty of Mechanical Engineering of Opole University of Technology. Currently, he is employed at the Department of Environmental Engineering at the Faculty of Mechanical Engineering of Opole University of Technology and is enrolled at $\mathrm{PhD}$ studies. His scientific interests focus on IC engines, with a particular emphasis on the topic of air flow in the engine intake systems. He specializes in the use of visual methods commonly employed in Environmental Engineering to study and research air flow in engine intake systems. 
